\title{
CNTO6785, a Fully Human Antiinterleukin 17 Monoclonal Antibody, in Patients with Rheumatoid Arthritis with Inadequate Response to Methotrexate: A Randomized, Placebo-controlled, Phase II, Dose-ranging Study
}

Philip J. Mease, Slawomir Jeka, Juan Jose Jaller, Tasanee Kitumnuaypong, Worawit Louthrenoo, Herman Mann, Galina Matsievskaia, Enrique R. Soriano, Bin Jia, Caihong Wang, Jing Nie, and Elizabeth Hsia

ABSTRACT. Objective. To evaluate the efficacy, safety, pharmacokinetics, and immunogenicity of CNTO6785, a fully human monoclonal antibody that binds to human interleukin $17 \mathrm{~A}$, in patients with active rheumatoid arthritis (RA) with inadequate response to methotrexate (MTX) therapy.

Methods. This randomized, double-blind, placebo-controlled, dose-ranging study enrolled patients aged 18 to 80 years (inclusive) with active RA ( $\geq 6 / 66$ swollen and $\geq 6 / 68$ tender joints) who were refractory to MTX treatment (7.5-25 mg weekly, inclusive). The study duration was 38 weeks, containing a 10-week safety followup. Patients were randomized 1:1:1:1:1 to receive CNTO6785 15, 50,100 , or $200 \mathrm{mg}$ every 4 weeks + MTX or placebo + MTX. The primary endpoint was American College of Rheumatology 20 (ACR20) response at Week 16.

Results. There were no significant differences from placebo in the proportion of patients treated with CNTO6785 in the primary endpoint of ACR20 response at Week 16. There were no significant findings in any additional efficacy variables through Week 32. No dose-response relationships or specific patterns were observed in adverse event profiles among CNTO6785 treatment groups. Infections occurred with similar frequency across all groups, and injection site reactions were mild or moderate and did not demonstrate a dose-response relationship. Median serum CNTO6785 concentration increases through Week 38 were about dose-proportional; the incidence of neutralizing antidrug antibodies was $19.4 \%$ and was not associated with study drug dose level.

Conclusion. CNTO6785 was well tolerated, but did not demonstrate clinical efficacy in patients with active RA with inadequate response to MTX. (First Release November 1 2017; J Rheumatol 2018;45:22-31; doi:10.3899/jrheum.161238)

\section{Key Indexing Terms:}

INTERLEUKIN 17

METHOTREXATE

\section{RHEUMATOID ARTHRITIS} CLINICAL TRIAL, PHASE II

P.J. Mease, MD, Swedish Medical Center and University of Washington; S. Jeka, Professor, MD, Department of Rheumatology and Connective Tissue Diseases, 2nd University Hospital, CM UMK; J.J. Jaller, MD, Scientific Director, Centro de Reumatologia y Ortopedia, Docente Investigador, Universidad Metropolitana; T. Kitumnuaypong, MD, Rajavithi Hospital; W. Louthrenoo, MD, Division of Rheumatology, Department of Internal Medicine, Faculty of Medicine, Chiang Mai University; H. Mann, MD, Institute of Rheumatology; G. Matsievskaia, $M D$, Chief of Rheumatology Department, Clinical Rheumatology Hospital \#25; E.R. Soriano, MD, MSc, Head Rheumatology Unit, Hospital Italiano de Buenos Aires; B. Jia, PhD, Janssen (China) Research and Development Center; C. Wang, MD, Immunology Therapeutic Area Head, Janssen (China) Research and Development Center; J. Nie, PhD, Senior Manager, Clinical Pharmacology, Janssen (China) Research and Development Center; E. Hsia, MD, MSCE, Janssen Research and Development LLC. Address correspondence to Dr. P.J. Mease, Seattle Rheumatology Associates, 601 Broadway, Suite 600, Seattle, Washington 98102, USA. E-mail:pmease@philipmease.com

Full Release Article. For details see Reprints and Permissions at jrheum.org Accepted for publication July 28, 2017. $J$. Nie are full-time employees of Janssen (China) Research and Development Center. E. Hsia is a full-time employee and stockholder of Janssen Research and Development LLC. 
Rheumatoid arthritis (RA) is characterized by the immune-mediated destruction of joints, largely mediated by T cells and inflammatory cytokines ${ }^{1}$. Disease-modifying antirheumatic drugs (DMARD) are the recommended first-line therapy for $\mathrm{RA}^{2}$, but do not induce adequate response in a substantial number of patients and are associated with considerable toxicity. Hence, there is an unmet need for clinically efficacious therapies in $\mathrm{RA}^{3}$. New treatment strategies using combinations of methotrexate (MTX) and biologic agents targeting immune components such as tumor necrosis factor- $\alpha$ (TNF- $\alpha$ ) have made clinical remission, low disease activity, and structural and functional remission a realistic goal for patients with RA ${ }^{1}$.

Interleukin 17A (IL-17A) is one of several immune mediators that may contribute to the pathogenesis of RA. Under normal conditions, IL-17 levels are extremely low or undetectable in human sera; however, studies have shown elevated IL-17 levels in sera and synovial fluid in a subset of patients with $\mathrm{RA}^{4,5}$. Moreover, immunohistochemical staining of RA synovium has identified a subset of infiltrating T cells expressing IL-176,7. Blocking the biologic activity of IL-17 may be beneficial in RA treatment ${ }^{8}$, potentially reducing joint inflammation and preventing bone erosion ${ }^{9}$.

CNTO6785 is a fully human immunoglobulin G1 $\lambda$ monoclonal antibody (mAb) that binds to human IL-17A with high affinity and specificity, but does not bind to IL-17F. CNTO6785 inhibits the binding of IL-17A to its receptor, and in in vitro assays using primary human cells, has been shown to inhibit the biological activity of both human recombinant and native IL-17A. In vitro studies have shown that CNTO6785 binds to and inhibits the biological activity of cynomolgus monkey IL-17A with comparable affinity and activity to human IL-17A.

The primary objective of our study was to evaluate the efficacy of CNTO6785 for improving signs and symptoms of active RA despite concomitant MTX therapy. This study was also designed to evaluate the effects of CNTO6785 on physical function, safety, tolerability, pharmacokinetics (PK), and immunogenicity of CNTO6785 in this population.

\section{MATERIALS AND METHODS}

This was a randomized, double-blind, placebo-controlled, dose-ranging study conducted at multiple sites in Europe, Asia, and Latin America, in accordance with ethical principles of the Declaration of Helsinki and consistent with Good Clinical Practices and applicable regulatory requirements. The study protocol was approved by the independent ethics committees at the study centers of the authors (details of approval, including ethics committee name and approval number, are provided in Supplementary Table 1, available with the online version of this article). The study protocol was also approved by the independent ethics committee or institutional review board at each additional participating center (complete list in Supplementary Table 2, available with the online version of this article).

Patients. Males or females aged 18 to 80 years (inclusive) with a diagnosis of RA for $\geq 6$ months, per revised 1987 criteria of the American Rheumatism Association, were eligible for inclusion ${ }^{10}$. The study enrolled patients with active disease, defined as $\geq 6$ swollen and $\geq 6$ tender joints using a 66/68 joint count at screening and baseline. Serum C-reactive protein $(\mathrm{CRP}) \geq 0.8$ $\mathrm{mg} / \mathrm{dl}$ at screening or erythrocyte sedimentation rate $\geq 28 \mathrm{~mm}$ in the first hour at screening or baseline was also required. Patients were treated with and tolerated MTX at doses from $7.5 \mathrm{mg} /$ week to $25 \mathrm{mg} /$ week (inclusive) for $\geq 6$ months before screening and were receiving a stable MTX dose for $\geq 6$ weeks before the first study agent administration. Use of nonsteroidal antiinflammatory drugs (NSAID) or other analgesics must have been stable for $\geq 2$ weeks before the first study agent administration, and use of oral corticosteroids ( $\leq 10 \mathrm{mg} /$ day prednisone or equivalent) must have been stable for $\geq 4$ weeks prior to the first study agent administration. Oral corticosteroids, NSAID, or other analgesics within 2 weeks before the first study agent administration were not permitted for patients not using them at randomization. Patients were ineligible to participate if they had a history of any inflammatory disease other than RA, prior exposure to biologic therapy or nonbiologic DMARD other than MTX in the specified time frame, historical or ongoing malignancy within the prior 5 years (except for squamous and basal cell carcinomas of the skin or cervical cancer in situ treated with no evidence of recurrence), or infections.

Study design. The study design is summarized in Supplementary Figure 1 (available with the online version of this article). Following randomization, patients were followed for 38 weeks. Efficacy, safety, PK, and immunogenicity evaluations were performed on a prescribed schedule. During Week -6 to Week 0 , patients were screened to assess eligibility for enrollment, then randomized to 1 of 5 treatment groups and treated by subcutaneous (SC) administrations of study agent from Week 0 to Week 28. Patients in the control group received placebo every 4 weeks ( $\mathrm{q} 4 \mathrm{w}$ ) through Week 12, then crossed over to receive CNTO6785 $200 \mathrm{mg} \mathrm{q4w}$ from Week 16 to Week 28. The remaining groups initiated and continued to receive a consistent dose of $15 \mathrm{mg}, 50 \mathrm{mg}, 100 \mathrm{mg}$, or $200 \mathrm{mg}$ CNTO6785 q4w through Week 28. At each visit that required study agent administration, patients were administered $2 \mathrm{SC}$ injections each containing $2 \mathrm{ml}$ of study agent to maintain the blind. Week 28 to Week 38 consisted of the safety followup for all enrolled patients.

Evaluations. The primary efficacy endpoint was the proportion of patients demonstrating American College of Rheumatology 20 (ACR20) response ${ }^{11}$ at Week 16. Major secondary endpoints were ACR50 response at Week 16 and the change from baseline in the Disease Activity Score at 28 joints (DAS28) using CRP (DAS28-CRP) at Week 16. Additional secondary endpoints included the proportion of ACR20, ACR50, and ACR70 responders through Week 32; change from baseline at weeks 16 and 32 in DAS28, Clinical Disease Activity Index (CDAI), Simplified Disease Activity Index (SDAI), Health Assessment Questionnaire-Disability Index (HAQ-DI; Week 32 only), and Medical Outcomes Study Short Form-36 (SF-36); the proportion of patients in DAS28-CRP response (through Week 32) and remission (weeks 16 and 32); and the proportion of patients with ACR/European League Against Rheumatism (EULAR) remission using SDAI and Boolean criteria at weeks 16 and 32. Adverse events (AE), clinical laboratory tests (hematology, serum chemistry, and urinalysis), vital sign measurements, physical examinations, and electrocardiograms were monitored through Week 38.

For PK assessments, blood samples were collected at weeks 0, 2, 4, 8, $16,24,28,32$, and 38 from all randomized patients and serum CNTO6785 concentrations were analyzed using a validated electrochemiluminescent (ECL) immunoassay method on the Meso Scale Discovery (MSD) platform with a lowest quantifiable concentration of $0.16 \mu \mathrm{g} / \mathrm{ml}$. Pre-dose samples were collected at each dosing visit, and 1 random CNTO6785 concentration sample was collected between days 4 and 10 of Week 0 . For immunogenicity assessments, blood samples were collected from all randomized patients at weeks $0,16,24,28,32$, and 38 for the measurement of antibodies against CNTO6785, which were detected using a bridging ECL-based immunoassay (ECLIA) on the MSD platform. The results from the analysis of antidrug antibodies against CNTO6785 were classified as positive in patients with negative baseline samples and $\geq 1$ positive sample at any post-administration timepoint, or in patients with baseline positive samples and $\geq 1$ positive post-administration sample, plus a titer increase of $\geq 2$-fold after drug administration. Patients were classified as negative for antidrug antibodies if no

Personal non-commercial use only. The Journal of Rheumatology Copyright @ 2018 . All rights reserved. 
positive samples were detected at any post-administration timepoint. A validated CNTO6785-specific, ECL-based, competitive ligand-binding assay was used to identify neutralizing antibody (NAb)-positive patients among those who tested positive for antibodies to CNTO6785 by the ECLIA method. Antidrug antibodies-positive patients with any post-administration antidrug antibodies-positive samples that were NAb-positive were considered positive for NAb.

Statistical analyses. Primary and secondary efficacy analyses were based on the modified intent-to-treat population, defined as randomized patients who received $\geq 1$ partial dose of study agent. The safety analysis set included patients who received $\geq 1$ partial dose of CNTO6785 or placebo.

ACR20 response rates were analyzed using the Cochran-Mantel-Haenszel $(\mathrm{CMH})$ test stratified by region at a 2 -sided $10 \%$ significance level. Patients who met any of the following treatment failure criteria prior to Week 32 were considered nonresponders: initiated or increased the dose from baseline of oral corticosteroids or received intravenous or intramuscular corticosteroids for RA; increased baseline dose of MTX for RA; initiated treatment with DMARD, systemic immunosuppressives, and/or biologics for RA; or discontinued the study agent administration because of unsatisfactory therapeutic effect.

For secondary efficacy endpoints and demographic and baseline characteristics, descriptive statistics were used to summarize continuous variables; percentages were calculated for categorical variables. A 2-sided $\mathrm{CMH}$ test, logistic regression, or chi-square test was used for between-group comparisons of the proportion of patients achieving selected categorical endpoints A 2-sided ANOVA or ANCOVA was used for between-group comparisons for continuous endpoints. Safety endpoints were summarized using descriptive summary statistics. For PK analyses, serum CNTO6785 concentrations were summarized over time using descriptive statistics.

The required sample size was determined assuming that ACR20 response rate to placebo at Week 16 would be $35 \%$. A treatment difference of $25 \%$ in ACR20 response rate was considered for active treatment versus placebo. With these assumptions, 50 patients in the placebo group and each of the CNTO6785 groups ensured about $80 \%$ power at a 1 -sided significance level of 0.05 for detecting a difference when comparing a CNTO6785 treatment group with the placebo group, without multiplicity adjustment.

\section{RESULTS}

Patients. Baseline patient characteristics were generally similar and well balanced between treatment groups (Table 1). The median duration for RA was 4.8 years for the total population, 5.4 years in the placebo group, and 4.75 years in the combined CNTO6785 group (Table 1). All patients were using MTX at baseline; 105 patients (51\%) were treated with MTX for $>3$ years, with a median dose of $15 \mathrm{mg} /$ week through Week 38. Patient disposition is provided in Supplementary Figure 2 (available with the online version of this article).

Efficacy. For the primary efficacy endpoint, there were no statistically significant differences in the proportion of patients who achieved ACR20 response at Week 16 between the placebo group and any of the individual or combined CNTO6785 treatment groups (Figure 1A). In addition, no dose-response relationship of CNTO6785 was observed. Similarly, no treatment difference between any CNTO6785 group and the placebo group was observed in subgroup analyses by region. ACR20 response rates at Week 16 for the combined CNTO6785 group versus placebo were $44.4 \%$ versus $42.9 \%$ in Asia, $39.0 \%$ versus $33.3 \%$ in Europe, and $55.4 \%$ versus $57.1 \%$ in Latin America.
The proportions of responders over time are shown for ACR20 (Figure 1B), ACR50 (Figure 1C), and ACR70 (Figure 1D). No notable differences were observed in the proportions of responders when comparing the placebo group with any CNTO6785 treatment groups through Week 32; however, corresponding values were numerically higher at Week 32 compared with Week 16. No dose-response relationship was observed in any of the CNTO6785 treatment groups in ACR20, ACR50, or ACR70 through Week 32.

There were no significant differences between the placebo and CNTO6785 treatment groups in change from baseline in DAS28-CRP through Week 32 (Figure 2A) or in the proportion of patients with DAS28-CRP response (data not shown). The proportion of patients in DAS28-CRP remission at Week 16 was numerically higher with CNTO6785 (combined group, $15.0 \%$ ) compared with placebo (7.8\%). No consistent dose-response relationship was observed between the CNTO6785 groups for DAS28-CRP response or remission. The proportion of patients achieving SDAI-based ACR/EULAR remission was 3.9\% with placebo and $4.9 \%$ with CNTO6785 (combined group) at Week 16, while at Week 32 corresponding values were $7.8 \%$ and $12.5 \%$, respectively. The proportion of patients who achieved Boolean-based ACR/EULAR remission was $3.9 \%$ for placebo at both weeks 16 and 32 and $4.4 \%$ and $7.8 \%$ in the combined CNTO6785 group at weeks 16 and 32, respectively. These differences were not statistically significant. There were no significant differences between the placebo and CNTO6785 groups in mean change from baseline in CDAI and SDAI (data not shown).

There was a noticeable trend of decreased CRP level through Week 32 with CNTO6785 treatment; however, the change was limited, with no detectable dose-response relationship (Figure 2B). At Week 16, there was no significant difference in mean change from baseline between placebo $(-0.03 \mathrm{mg} / \mathrm{dl})$ and any of the CNTO6785 groups $(-0.82 \mathrm{mg} / \mathrm{dl},-0.34 \mathrm{mg} / \mathrm{dl},-0.40 \mathrm{mg} / \mathrm{dl}$, and $-0.76 \mathrm{mg} / \mathrm{dl}$ in the CNTO6785 $15 \mathrm{mg}, 50 \mathrm{mg}, 100 \mathrm{mg}$, and $200 \mathrm{mg}$ groups, respectively) or the combined CNTO6785 group ( -0.58 $\mathrm{mg} / \mathrm{dl})$. Although changes from baseline in CRP were greater at Week 32 than Week 16 within groups, including a numerically greater decrease in the placebo group after switching to CNTO6785 at Week 16, there were no notable differences between treatment groups.

The HAQ-DI showed no significant differences between the placebo and CNTO6785 treatment groups through Week 32 , and no dose-response relationship was observed in the CNTO6785 groups (Figure 2C). Additionally, there were no significant differences between the placebo group and any CNTO6785 group(s) in mean improvements from baseline in the SF-36 mental and physical component summary scores (data not shown).

Safety. CNTO6785 was generally well tolerated for the 28 -week treatment at doses ranging from $15 \mathrm{mg}$ to $200 \mathrm{mg}$

Personal non-commercial use only. The Journal of Rheumatology Copyright (c) 2018. All rights reserved. 
Table 1. Patient baseline demographics and disease characteristics.

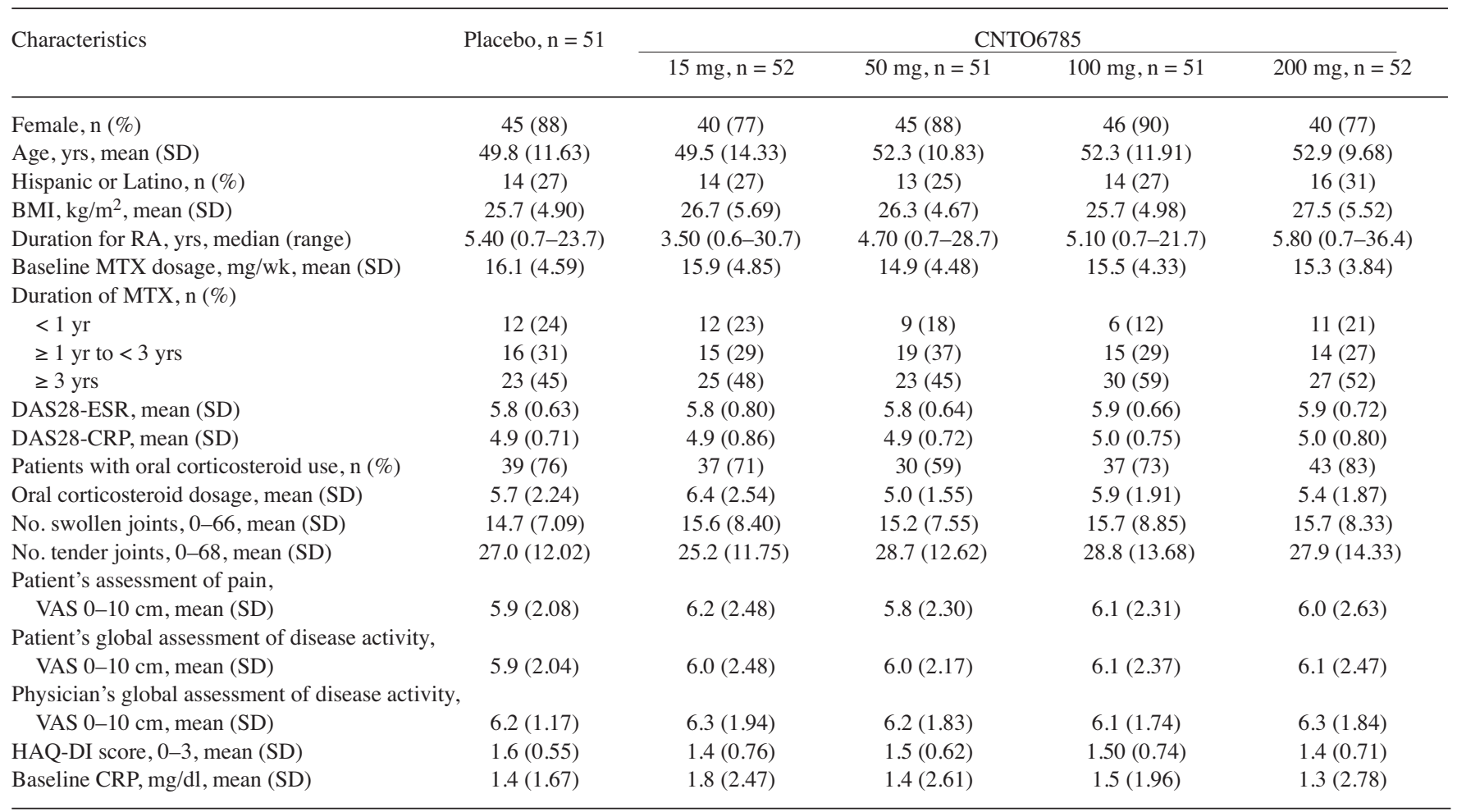

Percentages calculated with the number of patients in each treatment group as the denominator. Some variables had missing values and $\leq 5$ cases evenly occurred in each treatment group. BMI: body mass index; CRP: C-reactive protein; DAS28: Disease Activity Score at 28 joints; ESR: erythrocyte sedimentation rate; HAQ-DI: Health Assessment Questionnaire-Disability Index; MTX: methotrexate; RA: rheumatoid arthritis; VAS: visual analog scale.

q4w. No consistent dose-response relationships or specific patterns were observed in treatment-emergent AE (TEAE) profiles among individual CNTO6785 treatment groups (Table 2). Through Week 16, the proportion of patients with $\geq 1$ TEAE was higher in all CNTO6785 treatment groups compared with the placebo group. This was driven primarily by the higher occurrence of injection site reactions $(2.0 \%$ in the placebo group and 19.9\% in the combined CNTO6785 group). Other TEAE profiles in the CNTO6785 groups were similar to those observed in the placebo group.

Among AE of special interest, infections occurred with similar frequency across all treatment groups (Table 2). Two serious infections (community-acquired pneumonia) were reported. One occurred in a patient in the placebo-to-200-mg group and required hospitalization. This event occurred about 8 weeks after the last dose (Week 28) and was considered not related to the study agent. The other case was in the CNTO6785 100-mg group and resulted in the interruption of 1 dose of study agent. This event was considered probably related to the study agent and the patient recovered with appropriate treatment. Additionally, 1 opportunistic infection of ophthalmic herpes zoster occurred in the CNTO6785 15-mg group and was not considered related to the study agent; however, study treatment was discontinued. Through Week 38, no active tuberculosis or Candida infection was reported.
All injection site reactions were of mild or moderate intensity, did not demonstrate a dose-response relationship, and only 1 case (in the 100-mg group) resulted in withdrawal of the study agent. Injection site reactions were primarily injection site pain (placebo, 2.0\%; combined CNTO6785, $16.5 \%$ ) and injection site erythema (placebo, 0 ; combined CNTO6785, 3.9\%). In most cases, the first occurrence of injection site reaction was at the first SC injection. There was no apparent relationship between injection site reactions and development of antidrug antibodies. No clinically important, consistent differences were observed among/between treatment groups in laboratory safety monitoring tests through Week 38. More patients experienced decreased neutrophils or leukocytes in the combined CNTO6785 group (29 in Common Terminology Criteria for Adverse Events Grade 1, 6 in Grade 2, and 1 in Grade 4) compared with placebo (1 in Grade 1). For the patient with a Grade 4 TEAE, a test error was suspected; levels returned to normal during a test repeated shortly thereafter without any treatment. All neutrophil or leukocyte decreases were transient and asymptomatic, and did not lead to study agent discontinuation.

There was 1 case each of malignancy (breast cancer stage III) and major adverse cardiovascular event (cerebrovascular accident). Overall, no major safety signals were detected, including those related to depression or suicide risk.

Personal non-commercial use only. The Journal of Rheumatology Copyright @ 2018 . All rights reserved. 

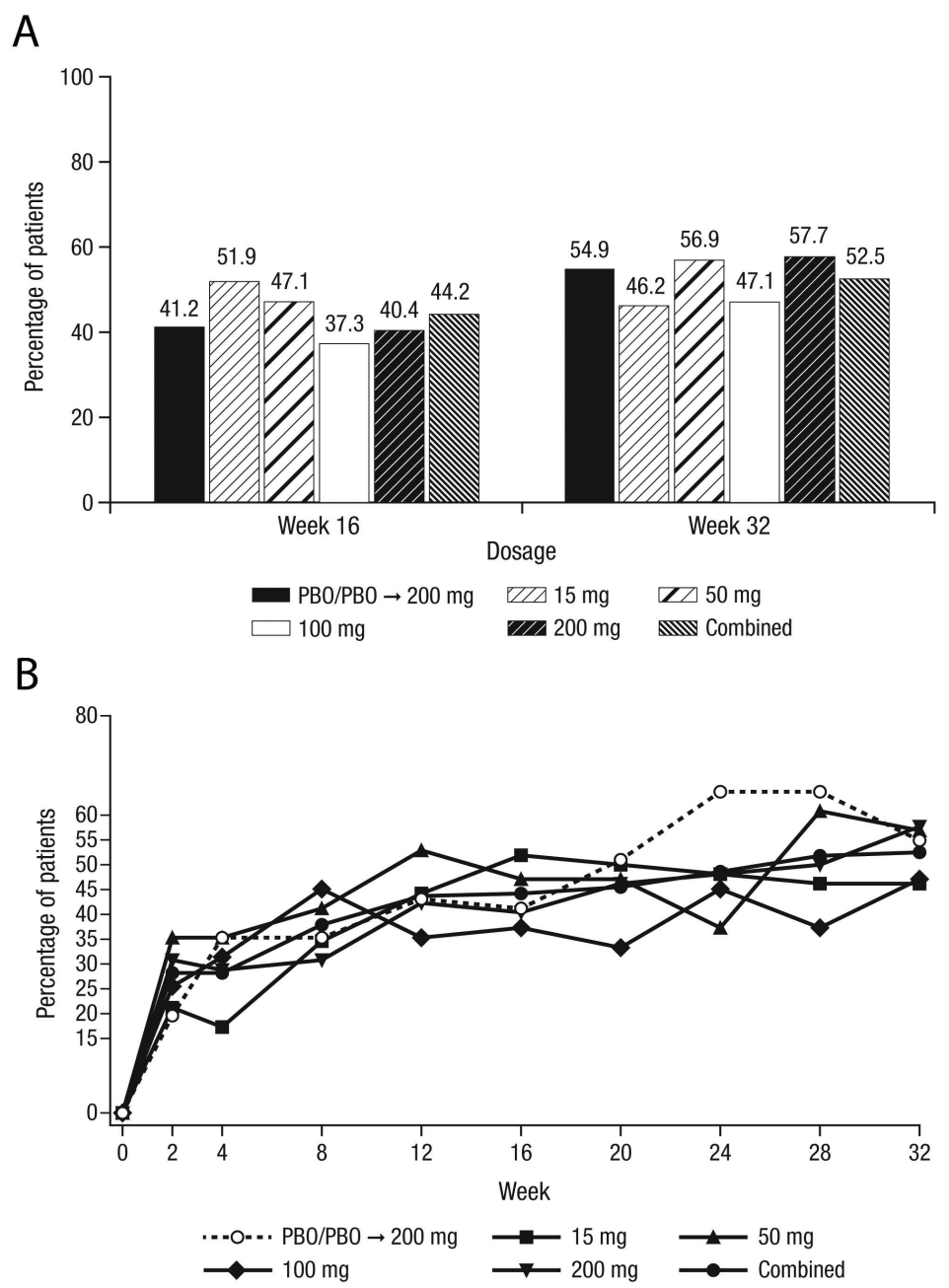

Figure 1. ACR results. (A) Primary endpoint: ACR20 at Week 16 (Week 32 is also shown). Secondary endpoints: proportions of patients who achieved an (B) ACR20, (C) ACR50, or (D) ACR70 response over time through Week 32. ACR: American College of Rheumatology; PBO: placebo.

PK/immunogenicity. Median serum CNTO6785 concentrations through Week 38 showed nearly dose-proportional increases after initial administration and following multiple administrations (Figure 3). Median trough concentrations at Week 24 and Week 28 were comparable between groups, suggesting that steady state was achieved by Week 24 . Beyond Week 24, average serum CNTO6785 concentrations in the placebo-to-200-mg group were not significantly different from those in the CNTO6785 200-mg group. The incidence of antidrug antibodies to CNTO6785 was $19.4 \%$ (48/247) through Week 38 (Table 3), and did not appear to be associated with dose level. Most antidrug antibody responses (96\% of patients) showed a peak titer value of $\leq 1: 320$, and all antidrug antibody responses were neutralizing to CNTO6785. Within the same dose level, serum CNTO6785 concentrations were generally lower in patients who tested positive for antidrug antibodies than those who tested negative after 8 to 16 weeks of treatment.

\section{DISCUSSION}

Our study was undertaken to evaluate the clinical efficacy, safety, PK, and immunogenicity of CNTO6785, an anti-IL-17A $\mathrm{mAb}$. Laboratory data suggest that CNTO6785 will bind to human IL-17A with high affinity and specificity in vitro (data not shown). Evidence suggests that IL-17A may contribute to the pathogenesis of $\mathrm{RA}^{4,5,6,7}$, supporting IL-17A as a viable target for RA treatment. Prior phase II studies investigating the IL-17A-neutralizing mAb secukinumab ${ }^{12}$ and ixekizumab ${ }^{8}$ revealed modest improvements in ACR20 and DAS28-CRP responses, whereas the anti-IL-17 receptor type A mAb brodalumab did not demonstrate clinical efficacy in patients with $\mathrm{RA}^{13}$. Consistent with these findings, our study did not demonstrate efficacy in reducing signs and symptoms of active RA.

The demographic and baseline disease characteristics of our study were balanced within expected ranges and in alignment with the target study population. The 16-week, 

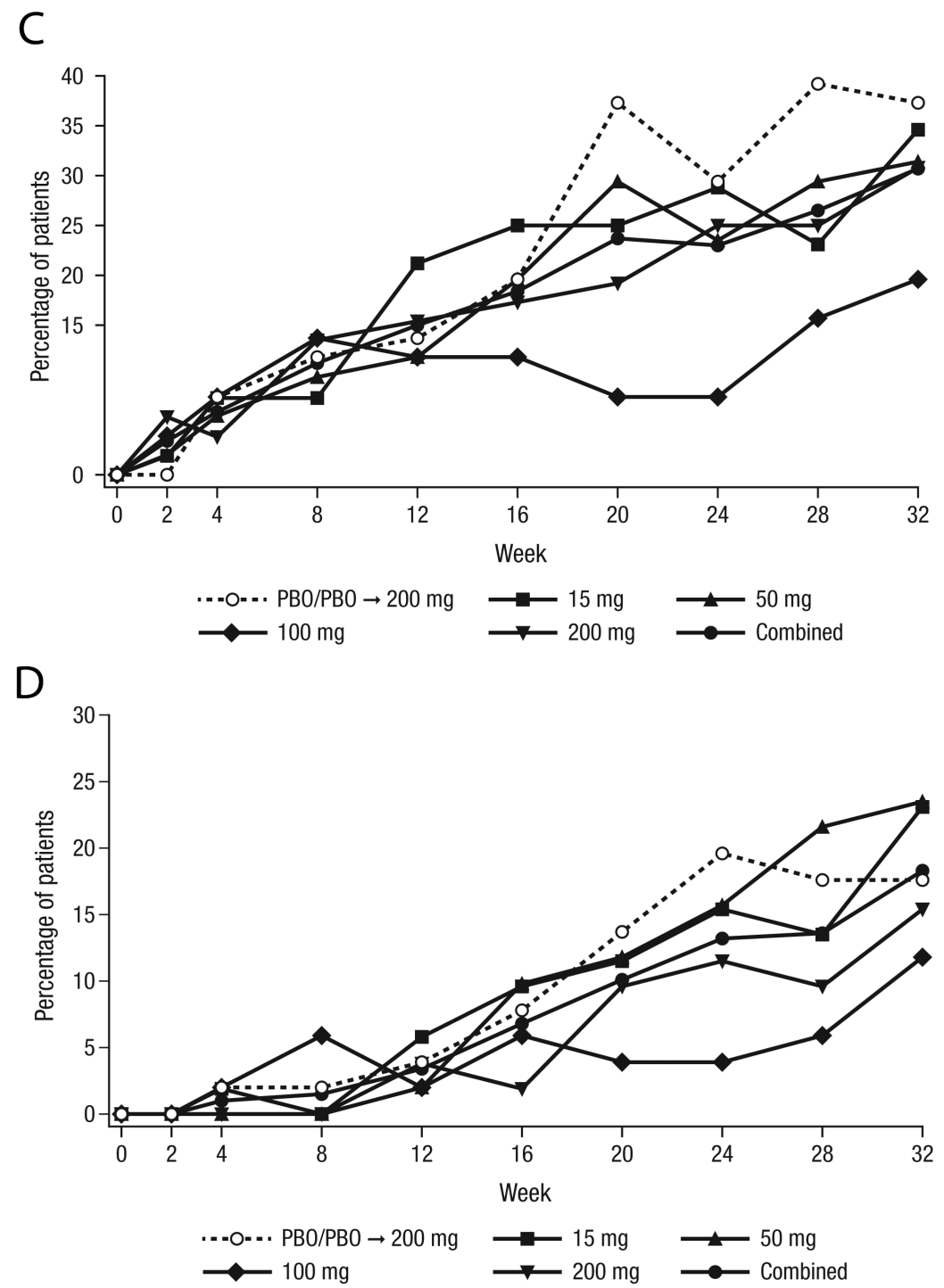

Figure 1. Continued.

placebo-controlled period is commonly seen in other similar trials, and is expected to be sufficient to see treatment differences between placebo and CNTO6785. Additionally, in our PK analysis, nearly dose-proportional increases in median serum CNTO6785 concentrations were observed, which is consistent with the first in-human study (CNTO6785OPD1001; data not shown) and within the expected range. The proportion of antidrug antibody-positive patients fell within a range expected for human therapeutic $\mathrm{mAb}$, and the titer of antidrug antibodies was generally low, suggesting little effect of antidrug antibodies on PK and clinical efficacy. Together, these findings suggest that drug exposure was adequate; thus, insufficient drug exposure was unlikely to explain the lack of significant efficacy differences between placebo and CNTO6785.

Another point to consider was the relatively high placebo effect $(41.2 \%)$ noted in our study, particularly in Latin
America (57.1\%). Although the reasons remain unclear, a high placebo effect was also observed in other phase II studies $8,12,14,15$. However, there were no significant efficacy differences between placebo and CNTO6785 [even in Europe where the placebo effect was lower (33.3\%)]; therefore, this is unlikely to be the reason for the negative results. Similarly, no treatment difference between any CNTO6785 group and the placebo group was observed in subgroup analyses by region (Asia, Europe, and Latin America). Therefore, ethnicity was not considered to have an effect on efficacy.

Numeric trends toward improvement were observed in studies of other anti-IL-17, suggesting some function of IL-17 in RA pathogenesis, but perhaps not as potent as other known factors such as TNF ${ }^{16}$ or IL- $6^{17}$. Although the change in CRP (a general marker of inflammation) was limited with no observed dose-response relationship, there was an overall trend of decreasing CRP levels through Week 32 with

Personal non-commercial use only. The Journal of Rheumatology Copyright @ 2018 . All rights reserved. 


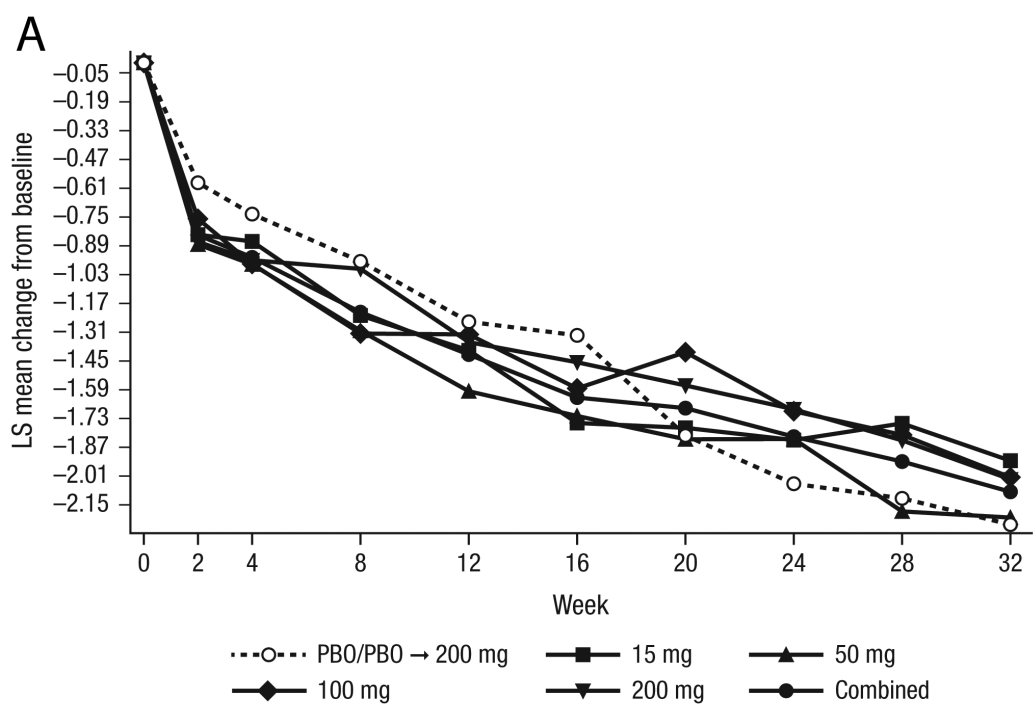

B

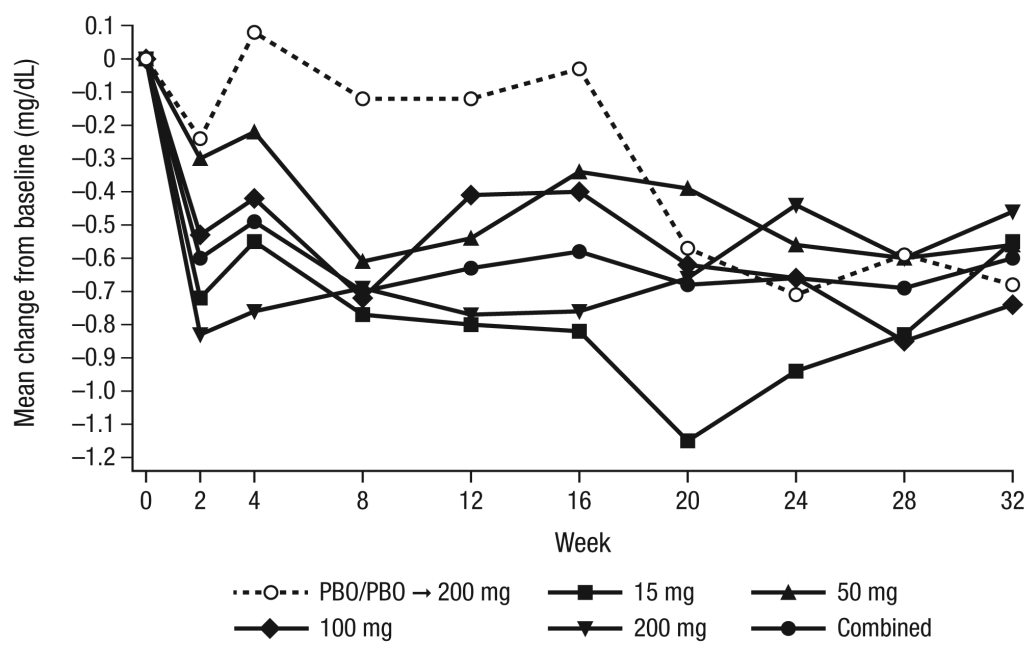

C

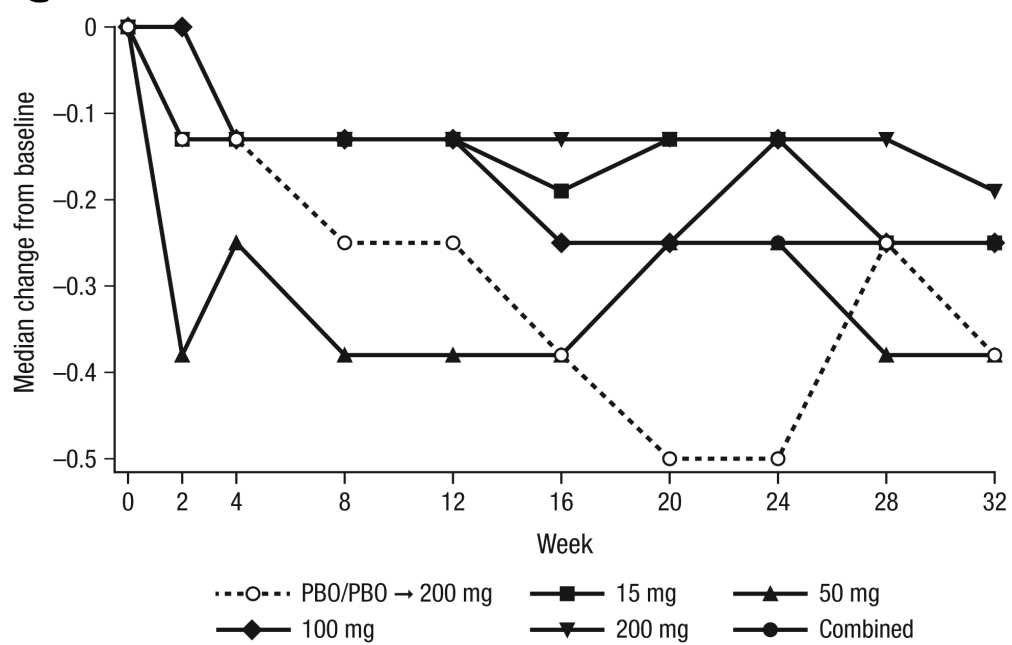

Figure 2. Change in secondary endpoints over time: (A) LS mean change from baseline in DAS28-CRP through Week 32, (B) mean change from baseline in CRP through Week 32, and (C) median change from baseline in HAQ-DI through Week 32. LS: least squares; DAS28: Disease Activity Score at 28 joints; CRP: C-reactive protein; HAQ-DI: Health Assessment Questionnaire-Disability Index; PBO: placebo. 
Table 2. Overall summary of TEAE through Week 38 .

\begin{tabular}{|c|c|c|c|c|c|c|}
\hline \multirow[t]{2}{*}{ Variables } & \multirow{2}{*}{$\begin{array}{l}\text { Placebo Wks } 0-16 \\
\qquad \mathrm{n}=51\end{array}$} & \multicolumn{5}{|c|}{ CNTO6785 } \\
\hline & & $\begin{array}{c}\text { Placebo to } 200 \mathrm{mg}, \\
\text { Wks } 16-38, \\
\mathrm{n}=48\end{array}$ & $\begin{array}{c}15 \mathrm{mg}, \\
\text { Wks } 0-38 \\
\mathrm{n}=52\end{array}$ & $\begin{array}{c}50 \mathrm{mg}, \\
\text { Wks } 0-38, \\
\mathrm{n}=51\end{array}$ & $\begin{array}{c}100 \mathrm{mg}, \\
\text { Wks } 0-38 \\
\mathrm{n}=51\end{array}$ & $\begin{array}{c}200 \mathrm{mg}, \\
\mathrm{Wks} 0-38, \\
\mathrm{n}=52\end{array}$ \\
\hline TEAE, n (\%) & $16(31.4)$ & $23(47.9)$ & $29(55.8)$ & $32(62.7)$ & $41(80.4)$ & $29(55.8)$ \\
\hline Treatment-emergent SAE, n (\%) & $1(2.0)$ & $1(2.1)$ & $2(3.8)$ & $2(3.9)$ & $5(9.8)$ & 0 \\
\hline \multicolumn{7}{|l|}{ Study agent discontinuation due } \\
\hline TEAE of interest, $\mathrm{n}(\%)$ & $10(19.6)$ & $19(39.6)$ & $22(42.3)$ & $27(52.9)$ & $28(54.9)$ & $21(40.4)$ \\
\hline Infections & $8(15.7)$ & $12(25.0)$ & $16(30.8)$ & $19(37.3)$ & $21(41.2)$ & $12(23.1)$ \\
\hline Injection site reactions & $1(2.0)$ & $8(16.7)$ & $9(17.3)$ & $13(25.5)$ & $12(23.5)$ & $10(19.2)$ \\
\hline Major adverse cardiovascular ever & vent & 0 & $1(1.9)$ & 0 & 0 & 0 \\
\hline Malignancies & 0 & 0 & 0 & $1(2.0)$ & 0 & 0 \\
\hline Neutropenia or leukopenia & $1(2.0)$ & $2(4.2)$ & $1(1.9)$ & $2(3.9)$ & $3(5.9)$ & $2(3.8)$ \\
\hline
\end{tabular}

TEAE of interest included neutropenia; decreases of leukocytes; infections including active tuberculosis, opportunistic infection, Candida infection, injection site reaction, newly identified malignancies, allergic reactions, major adverse cardiovascular events; and selected treatment-related SAE. TEAE: treatment-emergent adverse events; SAE: serious adverse events.

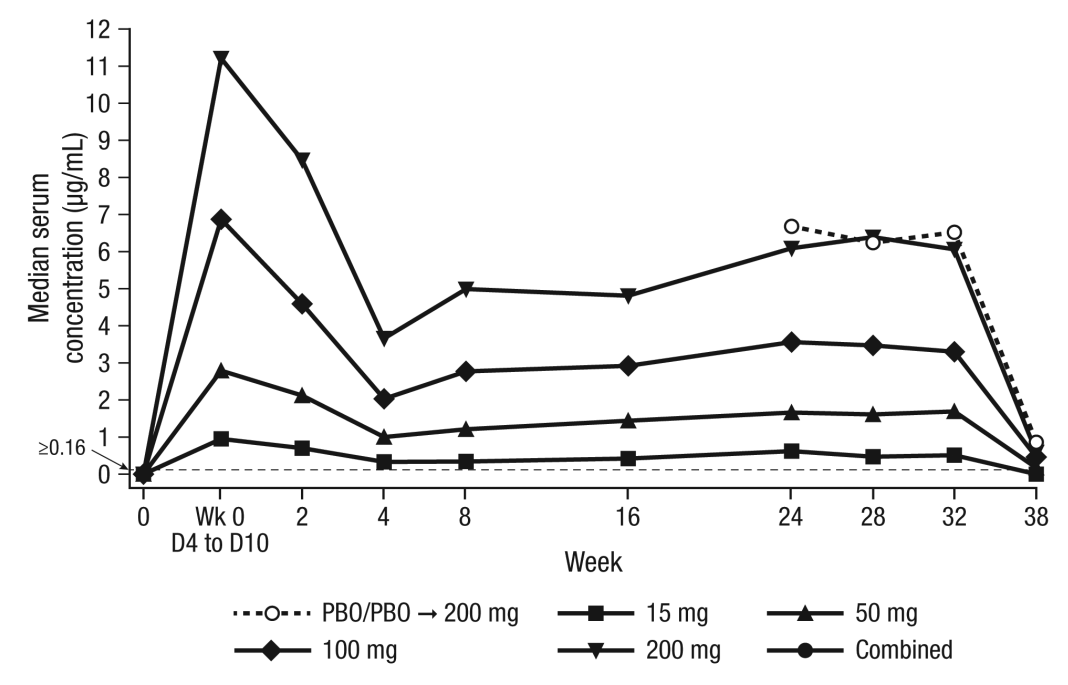

Figure 3. Median serum CNTO6785 concentration over time. PBO: placebo.

CNTO6785, suggesting CNTO6785 may partly inhibit inflammation in RA. Further, decreased neutrophil or leukocyte counts - a recognized specific effect of IL-17 blockers - were more frequent with CNTO6785 compared with placebo. These findings possibly reflect the target engagement of CNTO6785 in patients with RA.

The mechanisms of IL-17 are not fully elucidated. Although blocking the action of IL-17 as a single cytokine does not yield consistently robust results, the role of this pathway in the pathogenesis of RA may still warrant further investigation, potentially as a supplemental therapy target. Th17 cells express much higher levels of receptor activator of nuclear factor- $\kappa \mathrm{B}$ ligand (RANKL) than Th1 or Th2 cells, and thus may be efficient at promoting bone turnover ${ }^{18}$. IL-17-induced proteins, including matrix metalloproteinases, RANKL, and proinflammatory effectors such as inducible nitric oxide synthase, can cumulatively promote bone loss. It has been hypothesized that IL-17 blockade may have some effect on preventing bone erosion in RA and merits further analysis. There may be a benefit for anti-IL-17 agents in the treatment of early disease, or with advances in biomarker research, for use in selected patient populations ${ }^{19}$. Moreover, emerging studies have suggested that combined inhibition of targets, such as IL-17 and TNF, may work synergistically to increase the therapeutic response in $\mathrm{RA}^{20,21,22}$. In contrast, recently presented data on the bispecific compound ABT-122,

Personal non-commercial use only. The Journal of Rheumatology Copyright (C) 2018. All rights reserved. 
Table 3. Summary of antibodies to CNTO6785 status through Week 38.

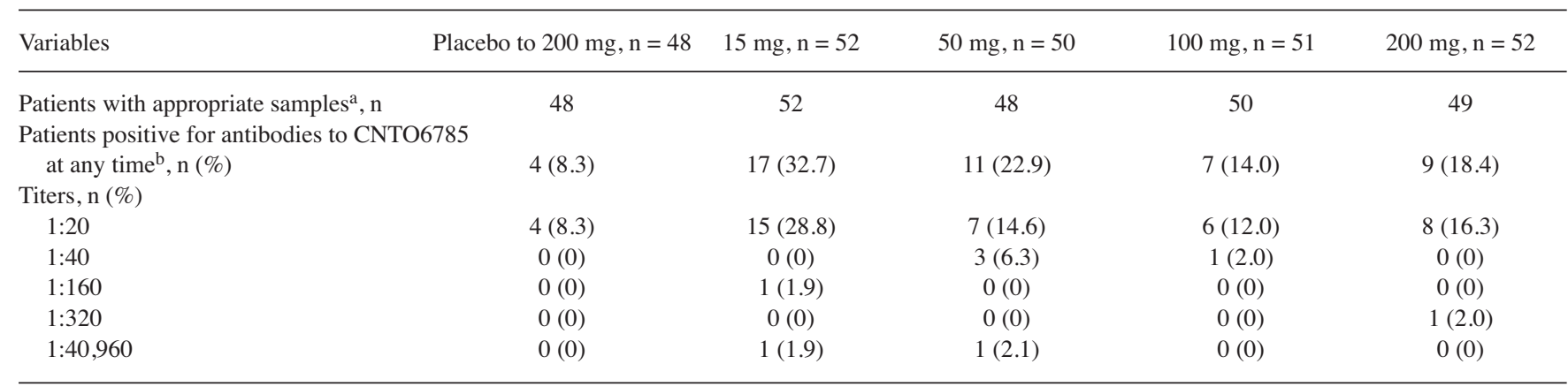

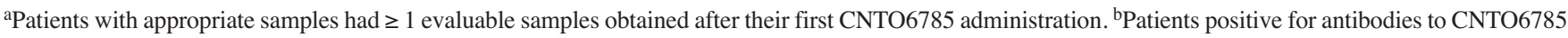
at any time are those who had $\geq 1$ sample that was positive for antibodies to CNTO6785 at any time. The denominator is the number of patients with appropriate samples. Patients with baseline positive samples, whereby the titer value did not increase more than 2-fold after CNTO6785 administration, were excluded.

which targets IL-17A and TNF, do not support synergistic efficacy ${ }^{23}$. Further studies will be needed to confirm or refute this hypothesis.

Despite the negative efficacy findings, CNTO6785 was well tolerated in patients with active RA. No major safety signals were detected for CNTO6785 given at doses up to $200 \mathrm{mg} \mathrm{q} 4 \mathrm{w}$ SC for 28 weeks. Overall, no consistent trends in safety signals were observed related to the dosing level throughout the study. This may be because the patient number was limited in each group. Further, the specified categories of TEAE of interest were generally balanced across the treatment groups, except injection site pain and erythema, which were identified as adverse drug reactions. The favorable safety profile of CNTO6785 suggests that it may be an appropriate candidate for indications in other autoimmune diseases.

\section{ACKNOWLEDGMENT}

We acknowledge all of the investigators who participated in the study: Joseph Antigua (Philippines), Caroline Arroyo (Philippines), Rosario Baes (Philippines), Dariusz Chudzik (Poland), Olga Ershova (Russia), Maria Victoria Garcia (Argentina), Joanna Hilt (Poland), Andrea Houzarova (Czech Republic), Krystyna Jedrychowicz-Rosiak (Poland), Larisa Knyazeva (Russia), Jana Kopackova (Czech Republic), Allan E. Lanzon (Philippines), Piotr Leszczynski (Poland), Pablo Jorge Maid (Argentina), Pablo Alejandro Mannucci (Argentina), Irina Marusenko (Russia), Maria Misterska-Skora (Poland), Sergey Noskov (Russia), Rodolfo Pardo Hidalgo (Argentina), Lucie Podrazilova (Czech Republic), Tatiana Popova (Russia), Tatiana Raskina (Russia), Mirhta Sabelli (Argentina), Evgeniya Shmidt (Russia), Mikhail Shostak (Russia), Svetlana Smakotina (Russia), Maria Rosa Ulla, (Argentina), Zuzana Urbanova (Czech Republic), Francisco Vargas (Colombia), Patricia Julieta Velez Sanchez (Colombia), and Agnieszka Zielinska (Poland).

\section{ONLINE SUPPLEMENT}

Supplementary material accompanies the online version of this article.

\section{REFERENCES}

1. Tanaka Y. Current concepts in the management of rheumatoid arthritis. Korean J Intern Med 2016;31:210-8.

2. Singh JA, Saag KG, Bridges SL Jr, Akl EA, Bannuru RR, Sullivan MC, et al. 2015 American College of Rheumatology guideline for the treatment of rheumatoid arthritis. Arthritis Rheumatol 2016;68:1-26.
3. Emery P, Klareskog L, Davis JC Jr, Westhovens R. Selective costimulation modulators: addressing unmet needs in rheumatoid arthritis management. MedGenMed 2005;6 Suppl:1.

4. Cho ML, Yoon CH, Hwang SY, Park MK, Min SY, Lee SH, et al. Effector function of type II collagen-stimulated T cells from rheumatoid arthritis patients: cross-talk between $\mathrm{T}$ cells and synovial fibroblasts. Arthritis Rheum 2004;50:776-84.

5. Ziolkowska M, Koc A, Luszczykiewicz G, Ksiezopolska-Pietrzak K, Klimczak E, Chwalinska-Sadowska H, et al. High levels of IL-17 in rheumatoid arthritis patients: IL-15 triggers in vitro IL-17 production via cyclosporin A-sensitive mechanism. J Immunol 2000;164:2832-8.

6. Chabaud M, Durand JM, Buchs N, Fossiez F, Page G, Frappart L, et al. Human interleukin-17: a T cell-derived proinflammatory cytokine produced by the rheumatoid synovium. Arthritis Rheum 1999;42:963-70.

7. Joosten LA, Radstake TR, Lubberts E, van den Bersselaar LA, Van Riel PL, van Lent PL, et al. Association of interleukin-18 expression with enhanced levels of both interleukin-1beta and tumor necrosis factor alpha in knee synovial tissue of patients with rheumatoid arthritis. Arthritis Rheum 2003;48:339-47.

8. Genovese MC, Durez P, Richards HB, Supronik J, Dokoupilova E, Aelion JA, et al. One-year efficacy and safety results of secukinumab in patients with rheumatoid arthritis: phase II, dose-finding, double-blind, randomized, placebo-controlled study. J Rheumatol 2014;41:414-21.

9. Genovese MC, Van den Bosch F, Roberson SA, Bojin S, Biagini IM, Ryan P, et al. LY2439821, a humanized anti-interleukin-17 monoclonal antibody, in the treatment of patients with rheumatoid arthritis: a phase I randomized, double-blind, placebo-controlled, proof-of-concept study. Arthritis Rheum 2010;62:929-39.

10. Arnett FC, Edworthy SM, Bloch DA, McShane DJ, Fries JF, Cooper NS, et al. The American Rheumatism Association 1987 revised criteria for the classification of rheumatoid arthritis. Arthritis Rheum 1988;31:315-24.

11. Felson DT, Anderson JJ, Boers M, Bombardier C, Chernoff M, Fried B, et al. The American College of Rheumatology preliminary core set of disease activity measures for rheumatoid arthritis clinical trials. The Committee on Outcome Measures in Rheumatoid Arthritis Clinical Trials. Arthritis Rheum 1993;36:729-40.

12. Genovese MC, Durez P, Richards HB, Supronik J, Dokoupilova E, Mazurov V, et al. Efficacy and safety of secukinumab in patients with rheumatoid arthritis: a phase II, dose-finding, double-blind, randomised, placebo controlled study. Ann Rheum Dis 2013;72:863-9. 
13. Martin DA, Churchill M, Flores-Suarez L, Cardiel MH, Wallace D, Martin R, et al. A phase Ib multiple ascending dose study evaluating safety, pharmacokinetics, and early clinical response of brodalumab, a human anti-IL-17R antibody, in methotrexate-resistant rheumatoid arthritis. Arthritis Res Ther 2013;15:R164.

14. Huizinga TW, Fleischmann RM, Jasson M, Radin AR, van Adelsberg J, Fiore S, et al. Sarilumab, a fully human monoclonal antibody against IL-6R $\alpha$ in patients with rheumatoid arthritis and an inadequate response to methotrexate: efficacy and safety results from the randomised SARIL-RA-MOBILITY Part A trial. Ann Rheum Dis 2014;73:1626-34.

15. Fleischmann R, Nayiager S, Louw I, Rojkovich B, Fu C, Udata C, et al. A multiple ascending dose/proof of concept study of ATN-103 (Ozoralizumab) in rheumatoid arthritis subjects on a background of methotrexate [abstract]. Arthritis Rheum 2011;63 Suppl 10:S1033.

16. Taylor PC, Feldmann M. Anti-TNF biologic agents: still the therapy of choice for rheumatoid arthritis. Nat Rev Rheumatol 2009; 5:578-82.

17. Kim GW, Lee NR, Pi RH, Lim YS, Lee YM, Lee JM, et al. IL-6 inhibitors for treatment of rheumatoid arthritis: past, present, and future. Arch Pharm Res 2015;38:575-84.
18. Gaffen SL. The role of interleukin-17 in the pathogenesis of rheumatoid arthritis. Curr Rheumatol Rep 2009;11:365-70.

19. Durez P. Is there a future for interleukin 17 blocking agents in rheumatoid arthritis? J Rheumatol 2016;43:465-7.

20. Buckland J. Rheumatoid arthritis: anti-TNF and anti-IL-17 antibodies-better together! Nat Rev Rheumatol 2014;10:699.

21. Taylor PC, Williams RO. Combination cytokine blockade: the way forward in therapy for rheumatoid arthritis? Arthritis Rheumatol 2015;67:14-6.

22. Fischer JA, Hueber AJ, Wilson S, Galm M, Baum W, Kitson C, et al. Combined inhibition of tumor necrosis factor $\alpha$ and interleukin-17 as a therapeutic opportunity in rheumatoid arthritis: development and characterization of a novel bispecific antibody. Arthritis Rheumatol 2015;67:51-62.

23. Georgantas RW III, Ruzek M, Davis JW, Hong F, Asque E, Idler K, et al. Genomic and epigenetic bioinformatics demonstrate dual TNF- $\alpha$ and IL17A target engagement by ABT-122, and suggest mainly TNF- $\alpha$-mediated relative target contribution to drug response in MTX-IR rheumatoid arthritis patients [abstract]. Arthritis Rheumatol 2016;68 Suppl 10:647. 